\title{
Erratum to: Thrombose de la veine mésentérique inférieure après gastrectomie en manchon
}

\section{Erratum to: Mesenteric venous thrombosis after laparoscopic sleeve gastrectomy}

\author{
P. Lointier $\cdot$ R. Kassir $\cdot$ C. Breton $\cdot$ T. Raia $\cdot$ P. Blanc
}

C) Springer-Verlag France 2013

(C) Springer-Verlag France 2013

Erratum to: Obésité DOI 10.1007/s11690-013-0390-9

Une erreur est intervenue concernant le titre de cet article : il fallait lire Thrombose de la veine mésentérique supérieure après gastrectomie en manchon

P. Lointier

Clinique de la Châtaigneraie,

59 , rue de la Châtaigneraie,

BP 125, 63110 Beaumont, Clermont-Ferrand

R. Kassir $\cdot$ C. Breton $\cdot$ T. Raia $\cdot$ P. Blanc $(\bowtie)$

Clinique Chirurgicale Mutualiste,

3, rue Le Verrier, BP 209, 42013 Saint-Etienne

e-mail : pblanc@mutualite-loire.com 